\title{
Hybrid Electrophototroph Enables High-Efficiency Carbon Dioxide Valorization to Fuel Molecules
}

\author{
Zhaodong Li \\ National Renewable Energy Laboratory \\ Chao Wu \\ National Renewable Energy Laboratory \\ Xiang Gao \\ National Renewable Energy Laboratory \\ Bennett Addison \\ National Renewable Energy Laboratory \\ Xihan Chen \\ National Renewable Energy Laboratory https://orcid.org/0000-0001-7907-2549 \\ Jianping Yu \\ National Renewable Energy Laboratory \\ Drazenka Svedruzic \\ National Renewable Energy Laboratory \\ Jeffrey Blackburn \\ National Renewable Energy Laboratory \\ Wei Xiong ( $\nabla$ Wei.Xiong@nrel.gov ) \\ National Renewable Energy Laboratory
}

\section{Article}

Keywords: Artificial Photosynthesis, Photosynthetic Electron Transfer Chain, Cyanobacteria, Bioelectrochemical Hybrid

Posted Date: October 30th, 2020

DOI: https://doi.org/10.21203/rs.3.rs-93987/v1

License: (c) (i) This work is licensed under a Creative Commons Attribution 4.0 International License. Read Full License 

Valorization to Fuel Molecules

3 Zhaodong $\mathrm{Li}^{1}$, Chao $\mathrm{Wu}^{2} \dagger$, Xiang $\mathrm{Gao}^{2} \dagger$, Bennett Addison ${ }^{2}$, Xihan $\mathrm{Chen}^{1}$, Jianping $\mathrm{Yu}^{2}$,

$5 \quad{ }^{1}$ Materials Science Center, National Renewable Energy Laboratory, Golden, CO.

$6 \quad{ }^{2}$ Biosciences Center, National Renewable Energy Laboratory, Golden, CO.

$7 \quad{ }^{*}$ Correspondence to: Jeffrey.Blackburn@nrel.gov and Wei.Xiong@nrel.gov

8 † Denotes equal contribution 


\section{Abstract}

13 Nature's biocatalytic processes are driven by photosynthesis, whereby photosystems I

14 and II are connected in series for light-stimulated generation of fuel products or electricity.

15 Externally supplying electricity directly to the photosynthetic electron transfer chain

16 (PETC) has numerous potential benefits, although strategies for achieving this goal have

17 remained elusive. Here we report an integrated photo-electrochemical architecture which

18 shuttles electrons directly to PETC in living cyanobacteria. The cathode of this

19 architecture electrochemically interfaces with cyanobacterial cells lacking photosystem II

20 activity that cannot perform photosynthesis independently. Illumination of the cathode

21 channels electrons from external circuit to intracellular PETC through photosystem I,

22 ultimately fueling $\mathrm{CO}_{2}$ conversion to acetate, a model fuel molecule with $9.32 \%$ energy

23 efficiency, exceeding the efficiency of natural photosynthesis in higher plants $(<1 \%)$ and

24 cyanobacteria ( 4-7\%). The resulting "Electrophototrophic" bio-electrochemical hybrid

25 has the potential to produce fuel chemicals with numerous advantages over standalone

26 natural and artificial photosynthetic approaches. 
Electrification of biocatalysis represents a strategic direction to meet global energy demand and foster development of efficient systems for conversion of $\mathrm{CO}_{2}$ to fuels and chemical feedstocks ${ }^{1-3}$. In nature, photosynthesis drives biocatalytic processes that form the basis of life on Earth by converting light energy to the chemical energy utilized for endergonic metabolism ${ }^{4}$. The central photosynthetic process involves coupled photoexcitation of two reaction center photosystems (PS) I and II (Fig. 1a). Excitation of PSI initiates electron transfer to ferredoxin and $\mathrm{NADPH}$ that energizes $\mathrm{CO}_{2}$-fixing pathways such as the Calvin-Benson-Bassham (CBB) cycle ${ }^{5}$. Photoexcitation of PSII results in oxygen evolution and electron transport from water to plastoquinone (PQ), which then regenerates neutral PSI through a series of reactions in the photosynthetic electron transfer chain (PETC) ${ }^{5}$.

Substantial research efforts have focused on leveraging biological photosystems (e.g. in algae and cyanobacteria) for sustainable production of energy products from sunlight ${ }^{6} 7$. For example, photosynthetic cyanobacteria have been engineered to produce fuel chemicals and polymers from $\mathrm{CO}_{2}{ }^{8-13}$. However, natural photosynthesis cannot use the full terrestrial solar irradiance, since photosynthetically active radiation (PAR) is limited to a subset of visible light (mostly 400-500 nm and 600-700 nm). Additionally, the natural photosynthetic $\mathrm{CO}_{2}$ fixation efficiency is diminished by the photorespiration process $^{14}$. Artificial photosynthetic solar-to-fuels cycles have been proposed as alternatives to natural photosynthesis ${ }^{15-17}$. These cycles can achieve high intrinsic energy efficiencies, but typically terminate at hydrogen and struggle to produce carbon-based biofuels at high energy and carbon conversion efficiencies ${ }^{18}$. We hypothesize that the ultimate goal of producing high-order carbon products at high energy efficiency may be achieved by interfacing natural and artificial photosynthesis in a hybrid system where a photosynthetic organism is synergistically energized by exogenous electrons through PETC. In the inverse of this process, photosynthetic fuel cells utilize "photo-electrogenic" microbes to generate electrical currents ${ }^{19-22}$. However, to date there is no integrated

60 electron transfer strategy that generates chemical energy upon external supply of 61 electricity to biological photosystems. 
Here we design, assemble, and optimize a self-sustained hybrid photosynthesis system that aims to circumvent limitations in natural photosynthesis and artificial solar fuels approaches. The crux involves electrochemical reactivation of a PETC-modified cyanobacterium with no PSII activity and cannot perform photosynthesis alone. We introduce a strategy and device to shuttle high-energy electrons into this cyanobacterium under light illumination, demonstrating $\mathrm{CO}_{2}$ conversion to fuel molecules such as acetate and amino acids ${ }^{23-25}$. Illuminating single photosystem (PSI) without the light absorption competition by the other (PSII) elevates efficiency ceiling of natural photosynthesis. The external electricity driving this reaction can be further harvested from multiple renewable sources, such as solar or wind, which are not limited by PAR and therefore enable a broader photosynthetic platform. This innovation introduces the concept of electrosynthetic cyanobacteria with the capability to drive carbon metabolism by both light energy and exogenous electricity. We describe this hybrid as an "electrophototrophic" system, a novel biotic-abiotic platform with the potential to valorize $\mathrm{CO}_{2}$ in higher energy conversion efficiency than natural photosynthesis, while producing more complex hydrocarbon fuels than artificial photosynthesis.

\section{A tailored photoelectrochemical system for electrophototrophy}

To energize photosynthesis via extracellular electron transport, we first blocked the natural photosynthesis pathway in the cyanobacterium where initial electrons are

82 generated from water splitting in PSII (Fig. 1a). This goal was achieved by either inhibiting

83 PSII activity physiologically via site-specific inhibitors ${ }^{26}$, or by leveraging a genetically generated PSII knockout mutant ${ }^{27}$. A mutant strain of the cyanobacterium Synechocystis sp. PCC 6803 (hereto Synechocystis), deficient in chlorophyll a binding protein (CP47) in PSII, cannot grow photoautotrophically. In the mutant (hereafter $\triangle \mathrm{PSII}$ ), PSII inactivity was shown by altered $77 \mathrm{~K}$ fluorescence spectrum (Fig. S1b) and significantly decreased 88 chlorophyll a level (Fig. S1c).

Next, we designed an electrochemical architecture (Fig. 1b) for exogenous electron delivery to cyanobacteria. This architecture allows physical attachment of

91 cyanobacterial cells to carbon felt and the transparent cathodic fluorine-doped tin oxide 
92 (FTO) electrode allows us to investigate light-activated photosystem driven by 93 extracellular electricity. FTO glass substrate faced-up porous carbon felt offers extremely

94 large interfacial area for bacteria loading (as shown in Fig. S2), excellent electron 95 transport properties, as well as short active species diffusion length for efficient 96 electrochemical reactions ${ }^{28}$.

\section{Light-dependent exogenous electron transfer to PETC}

Electrochemical devices for electrogenesis from photoautotrophically grown Synechocystis on anode were reported previously ${ }^{19,22,29}$. We first reproduced this electrogenesis process in our newly designed system and examined its electrochemical properties by interfacing biocompatible porous carbon felt with wild type (WT) Synechocystis cells (Fig. 1b). WT Synechocystis cells displayed a strong electrogenic response to chopped light (Fig. 2a left axis, red solid line), suggesting that physical contact between cells and the extracellular electron-transduction surface enables interfacial electron transfer. In contrast, no photocurrent was observed when applying anode potential ( $0.4 \mathrm{~V}$ vs. Ag/AgCl) to $\Delta$ PSII (Fig. 2a left panel, red dash line), consistent with previous reports that PSII is the primary source for electrogenesis ${ }^{21,29}$.

We next analyzed the properties of $\triangle \mathrm{PSII}$ as an electron acceptor by applying cathodic potential (-0.7 V vs. $\mathrm{Ag} / \mathrm{AgCl})$. Intriguingly, illumination of $\triangle \mathrm{PSIl}$ under cathodic 111 potential consistently increased cathodic current density (Fig. 2a left panel, black solid 112 line), whereas the WT Synechocystis which carries functional PSII did not produce a 113 photoelectrical response under cathodic bias (Fig. 2a left panel, black dash line). This 114 result implies that active PETC components downstream of PSII in the $\triangle P S I I$ mutant can 115 accept electrons from the external circuit in lieu of the deactivated PSII. Without redox 116 reactions by PSII, the light-dependent current response in $\triangle \mathrm{PSII}$ is in line with the photo117 reductive activity of PSI, the excitation of which can transfer electrons to the end of PETC, 118 thus allowing continuous electron input from external circuit. In contrast, photoexcited 119 PSII in the WT strain serves as the predominant electron donor, which could saturate the 120 PETC and diminish photoelectrical response significantly when injecting exogenous 121 electrons from cathode. 
We next use site-specific redox inhibitors to demonstrate that PETC components 123 downstream of PSII (see Fig. 1a) play a central role in electron flow from extracellular

124 circuit to cyanobacteria. Supplementation of the herbicide (3-3,4-dichlorophenyl)-1,1125 dimethylurea (DCMU), a specific inhibitor that blocks the binding site of $Q_{B}$ in the 126 photosystem ${ }^{30}$ (Fig. S3) did not diminish the light-dependent electrical response in $\triangle$ PSII 127 cells (Fig. 2b), suggesting that exogenous electrons can flow into the PETC downstream 128 of $Q_{B}$. Either blocking cytochrome b6f activity with 2,5-dibromo-3-methyl-6129 isopropylbenzoquinone (DBMIB) $)^{31}$ or inhibiting ferredoxin and NADP reduction with 130 phenylmercuric acetate (PMA) ${ }^{32}$ resulted in a significant decrease in photoelectrical 131 activity, as evinced by negligible changes of photocurrent density under light on and off 132 (Fig. 2b). These site-specific inhibitions support the mechanism that exogenous electrons 133 flow through cytochrome b6f, PSI and ultimately reach ferredoxin-NADP oxidoreductase

134 in the PETC. The opposing effect of DBMIB and DCMU further implies that PQ is probably 135 the entry point of exogenous electrons as it is the only PETC component between $Q_{B}$ and cytochrome b6f.

\section{Exogenous electrons energize $\mathrm{CO}_{2}$-to-fuels conversion with high energy efficiency} Motivated by global demand in $\mathrm{CO}_{2}$ recycling and energy production, we ask whether the exogenous electrons in our hybrid "electrophototrophic" system is able to 141 energize $\mathrm{CO}_{2}$ fixation and conversion to hydrocarbon fuels or fuel feedstocks. To answer 142 this question, we incubated $\triangle$ PSII cultures and applied electrical potential with 143 amperometric characterization. Light and electrical bias were systematically investigated 144 as two key variables, and we observe photosynthetic $\mathrm{CO}_{2}$ fixation and carbon product 145 formation only when supplying both illumination and exogenous electron supply (Fig. S4146 S6, more discussion in Supplementary Text). Shown in Fig. 3a, illumination on the 147 cathode (typical white LED for plant growth, $55 \mu \mathrm{mol} \mathrm{m} \mathrm{m}^{-2} \mathrm{~s}^{-1}$ on FTO glass) led to a 3.5148 fold increment of acetate production compared to its initial value with applied electrical 149 bias (-0.7 V vs. Ag/AgCl, intermittent supply, Fig. S4). In the dark, acetate concentrations

150 in the culture slightly decreased (from $\sim 270 \mu \mathrm{M}$ initial residual to $\sim 100 \mu \mathrm{M}$ ), presumably 151 due to non-photoexcited PSI which cannot reduce NADP and fuel carbon metabolism. 
152 The viability determined by optical density $\left(\mathrm{OD}_{730}\right)$ measurements indicate slight increase 153 under illumination, while the $\mathrm{OD}_{730}$ gradually declined $\sim 40 \%$ in dark after 8 days (Fig. S5).

154 In terms of exogenous electron supply, without negative electrical bias, no acetate 155 production was detected even though cells were illuminated constantly (Fig. S6).

156 As shown in Fig. 3b, acetate production by illuminated $\triangle \mathrm{PSII}$ was not found within 157 the first 5 days for application of either no bias or $-0.5 \mathrm{~V}$ vs. $\mathrm{Ag} / \mathrm{AgCl}$. In comparison, once 158 more negative bias (-0.7 V vs. Ag/AgCl, intermittent supply, Fig. S4) was applied (day 6159 10), acetate production resumed. Fig. S7 displays acetate yield as a function of various 160 potentials $(-0.15$ to $-0.7 \mathrm{~V})$ and indicates that potentials more negative than $-0.6 \mathrm{~V}$ vs. $161 \mathrm{Ag} / \mathrm{AgCl}$ can drive acetate production. Consistently, this threshold potential of $-0.6 \mathrm{~V}$ (vs. $162 \mathrm{Ag} / \mathrm{AgCl}$ ) is near the standard reducing potential of electrons in photoexcited PSII (Fig. 163 S7). This correlation implies a thermodynamic overpotential which could favor exogenous 164 electrons flowing into the bacteria downstream of PETC. Fig. $3 b$ demonstrates that 165 acetate concentration in medium increased steadily for 5 days during incubation under $1660.7 \mathrm{~V}$, eventually reaching $650 \mu \mathrm{M}$. Cell counts for $\Delta \mathrm{PSII}$, inferred by $\mathrm{OD}_{730}$ measurements, 167 decreased unless a certain bias was applied (Fig. S8). These results support our 168 hypothesis that the primary metabolic processes such as metabolite production and cell 169 maintenance can be energized by highly reductive exogenous electrons, flowing through 170 the PETC.

171 To further investigate the metabolic activities that can be driven by this 172 electrophototrophic system, we performed an isotope tracer analysis by adding ${ }^{13} \mathrm{C}$ 173 sodium bicarbonate into the $\triangle P S I I$ culture on cathode. Bicarbonate can be converted to $174 \mathrm{CO}_{2}$ by cyanobacterial carbonic anhydrase ${ }^{33}$. This $\mathrm{CO}_{2}$ can then drive carbon product 175 formation (acetate) and/or be fixed into biomass via cell metabolism. We first examined 176 the labeling fraction of acetate excreted into the medium. The GC-MS revealed the 177 production of ${ }^{13} \mathrm{C}$-acetic acid, indicating that newly fixed carbons end into this $\mathrm{C} 2$ product 178 (Fig. 3c). ${ }^{1} \mathrm{H}-\mathrm{NMR}$ spectra demonstrate that acetate was labeled in both methyl and 179 carboxyl carbons (Fig. S9) and allow us to evaluate the energy conversion efficiency in 180 the electro-photosynthetic process. Similar to the faradaic efficiency of conventional 181 electrochemical processes, the exogenous electrons involved in electrophototrophic 182 synthesis of acetate can be quantified by defining the exogenous electrons uptake 
183 efficiency (EEUE $E_{\text {acetate }}$ ). Over half $(61.8 \%)$ of exogenous electrons were utilized by $\triangle \mathrm{PSII}$

184 for selective acetic acid generation. Taking the incident photon flux into account, the 185 overall energy conversion efficiency is approximately $9.32 \%$ (see Supplementary Text 186 and Table S2). Even though this estimation only reflects the fixed carbons in acetate and 187 does not those fixed into biomass (vide infra), the value still exceeds typical natural energy 188 conversion efficiency of higher plants $(<1 \%)$ and cyanobacteria $(\sim 4-7 \%) .{ }^{34,35}$

We next analyzed the labeling patterns of seven proteinogenic amino acids that 190 are digested from cell biomass and are directly produced from the central carbon 191 metabolism (Fig. 3d). After four days incubation of $\triangle \mathrm{PSII}$ with ${ }^{13} \mathrm{C}$-bicarbonate under 192 constant white-light illumination, the cathodically biased cultures demonstrate partial ${ }^{13} \mathrm{C}$ 193 labeling in proteinogenic amino acids and display significantly higher fractional labeling 194 (FL, denoting the proportion of labeled carbons) than the negative control cultures without 195 applied bias. Serine, which can be synthesized from 3-phosphoglycerate, the first $\mathrm{CO}_{2}$ 196 fixation product of the CBB cycle, demonstrated a 3\% FL in comparison with $1 \%$ in the 197 negative control. This moderate ${ }^{13} \mathrm{C}$-accumulation is real because we indeed detected 198 significant increase of the $\mathrm{m}+1{ }^{13} \mathrm{C}$-pattern in the carboxylic group of serine, consistent 199 with the reaction skeleton of Ribulose-1,5-bisphosphate carboxylase/oxygenase 200 (RuBisCO) (Table S1). As another major $\mathrm{CO}_{2}$ entry point, ${ }^{13} \mathrm{C}$-bicarbonate can be fixed 201 by amphibolic reactions (e.g. phosphoenolpyruvate carboxylase) to generate 202 oxaloacetate which is the precursor of aspartate and threonine. Consistently, biased 203 cultures have much higher FL (7\%) in these two amino acids than those in the unbiased 204 cultures (1\%).

205 Interestingly, we observed a new $\mathrm{CO}_{2}$ fixation pathway activated in cyanobacteria 206 via glycine cleavage system which was found in Synechocystis ${ }^{36}$ but with no detailed in 207 vivo characterization. The metabolic activity of this $\mathrm{CO}_{2}$-fixing pathway can be reflected 208 by the extremely high fractional labeling in glycine over $30 \%$ when incubated $\triangle \mathrm{PSI}$ with $209{ }^{13} \mathrm{C}$-bicarbonate under constant white-light illumination for four days. Through this 210 pathway, $\mathrm{CO}_{2}$ will enter the one-carbon (C1) metabolism via formate which then forms 211 the methylene group of glycine. The GC-MS fragment of glycine (Gly_85) represents this 212 methylene group, demonstrating high FL (30\%) consistently. Our ${ }^{13} \mathrm{C}$-tracer analysis as 213 well as extracellular metabolite analysis support that exogenous electron supply to 
214 cyanobacterial PETC may lead to $\mathrm{CO}_{2}$ fixation and conversion, demonstrating a

215 functional bioenergetics system that fuels endergonic metabolism.

\section{Discussion}

218 This work provides electrochemical and biochemical evidence to support a proof219 of-concept hybrid electro-photosynthetic system that leverages exogenous electrons to 220 supplement photosynthetic energy conversion for driving $\mathrm{CO}_{2}$ fixation and conversion. 221 Cyanobacterial cells without PSII can sustain their metabolic viability on an electrode 222 surface and produce acetate, the primary excreting product (Fig. 3). Growing 223 photosystems-modified cyanobacteria in a photo-electrochemical architecture allows us 224 to expand the means by which photosynthetic organisms produce fuels and chemicals. 225 Such hybrid systems can access new pathways beyond canonical photosynthesis, the 226 inefficiency of which largely arises from the use of two photochemical systems with similar 227 absorption thresholds. The two photosystems (PSII, PSI) compete for the same regions 228 of the solar spectrum, cutting the energy efficiency nearly in half compared with what 229 might be achieved if the bandgaps were optimized to use different regions of the 230 spectrum ${ }^{35,37}$. From a broad standpoint, this work validates the first step of a new 231 photosynthesis concept to elevate the photosynthesis efficiency ceiling: Powering single 232 light-absorbing photosystem (PSI) via exogenous electricity which can be generated by 233 PV with extended range of solar energy absorption. Increases in efficiencies might be 234 obtained by PV devices that use the blue and near-UV region of the solar spectrum more 235 effectively or capture the energy of the sub-bandgap IR photons (illustrated in Fig. 4) ${ }^{35}$. 236 Such strategies may be promising for increasing the theoretical upper bound of natural 237 photosynthesis, as proposed by Blankenship and co-authors ${ }^{35}$.

238 Another merit for this hybrid photosynthesis approach arises from the fact that 239 inactivated PSIl does not evolve $\mathrm{O}_{2}$ as the photosynthetic byproduct. Suppressed $\mathrm{O}_{2}$ 240 evolution minimizes the propensity for RuBisCO to fix $\mathrm{O}_{2}$ as a competitive substrate for $241 \mathrm{CO}_{2}$. In natural photosynthesis, substrate competition initiates an energy-intensive 242 recovery process of photorespiration ${ }^{38}$ that can consume up to $25 \%$ of the initially stored 243 energy $^{39}$, a substantial source of inefficiency. Interestingly, although photorespiration also 
244 plays a biosynthetic role in metabolic processes, e.g. supplying glycine as an essential 245 metabolite $^{38}$, this role in hybrid photosynthesis seems to be substitutable with redundant 246 pathways, such as glycine cleavage system ${ }^{36}$. This notion is strongly supported by the 247 presence of pathway genes in cyanobacterial genome in line with isotope labeling 248 patterns as we provided here. Decrease in photorespiration thus underlie new 249 opportunities in the hybrid system to raise theoretical limits of photosynthesis.

250 More importantly, the hybrid system introduces a unique strategy for managing 251 photosynthetic outcomes. In natural photosynthesis, linear electron flow occurring 252 between two photochemical systems produces ATP and NADPH as energetic currency, 253 and their proportions are regulated for various biosynthetic purposes. Phototrophs 254 containing only PSI implement electron transport whereby electrons can be recycled from 255 either reduced ferredoxin or NADPH to $P Q$, and subsequently to the cytochrome b6f 256 complex $^{40}$. Such cyclic flow generates a pH gradient (and thus ATP), but without the 257 accumulation of reduced species for biosynthesis ${ }^{41}$. However, this study shows that 258 Synechocystis carrying single PSI can be electrically energized to fix $\mathrm{CO}_{2}$ and generate 259 building blocks of biomass, evinced by labeled proteinogenic amino acids from ${ }^{13} \mathrm{C}$ 260 bicarbonate. This study further indicates that the hybrid photo-electrochemical process 261 demonstrated here could enable on-demand control over the proportion of linear versus 262 cyclic electron flow to tailor the stoihiometric ratios of ATP and NADPH and ultimate 263 photosynthetic products. To achieve this goal, Nature evolved complicated regulatory 264 mechanisms to tune the ratio of PSI to PSII ${ }^{42,43}$. In the photo-electrochemical hybrid 265 demonstrated here, the ratios of energetic currency and products could instead be 266 regulated through the injection of exogenous electrons, which creates an artificial linear 267 electron flux that can be varied on-demand relative to cyclic electron flow by tuning the 268 cathodic current density and/or incident photon flux. Since this hybrid approach is not 269 tailored by evolution, it will be less constrained by the natural needs/environments to 270 implement. Instead, the hybrid can be optimized in well-designed conditions for targeted 271 ATP/NADPH ratio. Reengineering the system, for example on the biotic-abiotic interface, 272 is expected to improve overall efficiency for tunable electron transfer.

273 Taken together, the hybrid electrophototroph as we demonstrate, drives 274 exogenous electrochemical energy to replenish the universal energy and redox currency 
275 in living cyanobacteria for biosynthesis. Considering its functionality and a number of 276 advantages over pure natural/artificial photosynthesis, we posit that the development of

277 this bio-electrochemical platform will pave a new avenue to couple renewable electricity 278 with photobiological activities, a practical approach for production of hydrocarbon fuels 279 from sun and $\mathrm{CO}_{2}$.

\section{Methods}

282 Characterization of PSII knockout mutant in Synechocystis

The PSII deficient Synechocystis was a gift from Dr. Wim Vermaas at Arizona State 284 University. This mutant was generated by deleting the psbB gene which encodes 285 chlorophyll-binding protein CP-47 in PSII of Synechocystis. ${ }^{27}$ The slr0906 open reading 286 frame (ORF) encoding psbB was disrupted by inserting an antibiotic-resistance gene 287 cassette, replacing a part of the coding sequence. The genotype of the mutant was 288 verified by a PCR analysis using primers 0906_VF and 0906_VR (0906-VF: 289 CGTTACTAGAAGGAGCGTCA, 0906-VR: GGTACCTGGGGAGAGTAGAT). The $\triangle$ PSII 290 mutant and wild type Synechocystis were measured by fluorescence emission spectra 291 (77K) using a 435-nm excitation wavelength. The chlorophyll a level in the mutant was 292 quantitated after methanol extraction by measuring the absorbance of the supernatant at $293663 \mathrm{~nm}$, using glass cuvettes.

294 Cyanobacteria-electrode hybrid system

295 The PSII deficient Synechocystis ( $\Delta$ slr0906) was first inoculated and cultured 296 photoheterotrophically in BG11 medium with addition of $5 \mathrm{mM}$ glucose, under $30-50$ $297 \mu \mathrm{E} / \mathrm{m}^{2} / \mathrm{s}$ illumination at $30^{\circ} \mathrm{C}$. Exponentially growing cells were collected for further 298 applications.

299 In the following procedure, the tailored electrochemical $\mathrm{H}$-cell with three-electrode 300 configuration was applied for the electrochemical process. The reference and counter 301 electrodes were silver/silver chloride electrode and $\mathrm{Pt}$, respectively. The working 302 electrode and reference electrode ( $\mathrm{CH}$ Instruments, Inc.) were in the bottom chamber and 303 the Pt wire counter electrode was in the top chamber. A Nafion 117 membrane (Sigma- 
304 Aldrich) separates the two chambers. Each chamber has an inlet/outlet. The exponentially 305 growing culture was centrifuged, separated from the supernatant and re-dispersed in the medium (BG11+ bicarbonate, $\mathrm{pH}=7.8$ ). $\mathrm{A} \sim 7 \mathrm{ml}$ culture was transferred to the cathode chamber of the $\mathrm{H}$-cell, where the culture was illuminated from the bottom transparent 308 window. The device was air-tight and maintained at $30{ }^{\circ} \mathrm{C}$ for the duration of the electrochemical characterization.

310 Photoelectrochemical characterization

During the electrochemical incubation, a typical amperometry (i-t) procedure $(\mathrm{CH}$ 312 Instruments, Inc.) was conducted to check the ability of $\triangle P S I$ cyanobacteria as an 313 electron acceptor under illumination. It was conducted at different potentials ( $v s$. $\mathrm{Ag} / \mathrm{AgCl})$.

314 A $0.15 \mathrm{ml}$ culture was taken every day for $\mathrm{OD}_{730}$ and metabolite analysis.

315 PETC inhibition assay

Three PETC inhibitors: 3-(3,4-dichlorophenyl)-1,1-dimethylurea (DCMU), 2,5317 dibromomethylisopropyl-1,4-benzoquinone (DBMIB), and phenylmercury acetate (PMA) 318 were obtained from Sigma-Aldrich. They were dissolved in dimethyl sulfoxide for use. The working concentrations of DCMU, DBMIB and PMA were based on previous report ${ }^{21}$, 320 which were $2 \mu \mathrm{M}, 50 \mu \mathrm{M}, 200 \mu \mathrm{M}$, respectively. The photoelectrochemical measurements 321 were conducted after the inhibitors were supplemented into the culture for $10 \mathrm{~min}$.

322 Electron microscopy characterization

After the electrochemistry process, the carbon felt electrode was fixed in $2.5 \%$ 324 glutaraldehyde in phosphate buffer under $4{ }^{\circ} \mathrm{C}$ for $2 \mathrm{~h}$. The samples then underwent a 325 MilliQ water postfix wash and dehydration ( $24 \mathrm{~h}$ in a high vacuum desiccator). Scanning 326 Electron Microscopy (Hitachi S-4800 SEM) was applied to characterize the surface 327 morphology. Samples were imaged at $3 \mathrm{kV}$ acceleration, 7-10 $\mathrm{mm}$ working distance.

328 Quantitative analysis of acetate

We measured the excretion of acetate from Synechocystis using the following 330 method. The culture samples were collected and the supernatant was separated from 331 cells by filtration through $0.2 \mu \mathrm{M}$-diameter nylon membrane (Acrodisc $\circledast$ ). Acetate 332 concentration in each culture was analyzed with High Performance Liquid 
333 Chromatography (HPLC, Agilent Technologies 1200 series) by injecting $25 \mu \mathrm{L}$ samples 334 into an HPLC column (Bio-Rad Aminex HPX-87H), eluting with $5 \mathrm{mM}$ sulfuric acid at a 335 flow rate of $0.6 \mathrm{ml} / \mathrm{min}$, and detecting by a refractive index detector (retention time for 336 acetate: $15.2 \mathrm{~min})$. Standard samples with five different acetate concentrations $(2.5,5$, $33710,25$, and $50 \mathrm{mM})$ were used for quantification $\left(R^{2}=0.99839\right)$.

$338{ }^{13} \mathrm{C}$ - isotope tracer analysis to track carbon fixation

${ }^{13} \mathrm{C}$-bicarbonate was supplied during the electrochemical procedures to monitor 340 carbon metabolism in the photoelectrochemical environment. The ${ }^{13} \mathrm{C}$-labeled fraction of 341 acetate and protein-bound amino acids were measured by NMR and gas 342 chromatography-mass spectrometry (GC-MS), respectively. Exponentially growing $\triangle \mathrm{PSII}$ 343 cells were suspended in BG-11 medium supplemented with $100 \mathrm{mM}{ }^{13} \mathrm{C}$-labeled sodium 344 bicarbonate. The culture was applied in the electrochemical device under sunlight 345 simulated illumination (white LED, $55 \mu \mathrm{mol} \mathrm{m}^{-2} \mathrm{~s}^{-1} \mathrm{on}$ FTO glass). Cultures were sampled 346 at 0 hour, 2 day, 4 day and 5 day.

347 The sample treatment and GC-MS analysis were performed as previous 348 reported. ${ }^{44}$ Briefly, $5 \mathrm{~mL}$ of sampled cultures were centrifuged at $10,000 \mathrm{~g}$ for 1 minute, 349 the cell pellets were digested in $500 \mu \mathrm{L} 6 \mathrm{M} \mathrm{HCl}$ at $105^{\circ} \mathrm{C}$ for 12 hours. The hydrolysate 350 was dried under nitrogen gas flow at $65^{\circ} \mathrm{C}$, dissolved in $50 \mu \mathrm{L}$ water-free 351 dimethylformamide. For the GC-MS measurement the proteinogenic amino acids were 352 derivatized prior to analysis. The dried hydrolysate, dissolved in pyridine was derivatized 353 by $\mathrm{N}$-tert-butyldimethylsilyl-N-methyltrifluoroacetamide (TBDMS) with $1 \%$ tert-butyl354 dimethylchlorosilane at $85^{\circ} \mathrm{C}$ for $60 \mathrm{~min} .1 \mu \mathrm{L}$ of the sample in the organic phase was 355 loaded on the Agilent GC-6890 gas chromatography equipped with a Agilent 19091J-413 356 column $(30 \mathrm{~m} \times 0.32 \mathrm{~mm} \times 0.25 \mu \mathrm{m})$ directly connected to a MS-5975C mass spectrometer. 357 Helium was used as the carrier gas. The oven temperature was initially held at $50^{\circ} \mathrm{C}$ for $3582 \mathrm{~min}$; then raised to $150^{\circ} \mathrm{C}$ at $5^{\circ} \mathrm{C} / \mathrm{min}$ and held at that value for $2 \mathrm{~min}$; finally, it was 359 raised to $320^{\circ} \mathrm{C}$ at $7^{\circ} \mathrm{C} / \mathrm{min}$, and held at that final value for $2 \mathrm{~min}$. Other settings included 360 splitless and electron impact ionization (EI) at $70 \mathrm{eV}$. The FLs of alanine, aspartate, 361 glutamate, glycine, phenylalanine, serine, threonine was analyzed. 
To analyze the isotope labeling pattern of amino acids, a mass isotopomer distribution vector, $M D V_{\alpha}$, was assigned according to Nanchen et al ${ }^{45}$.

364

$$
\operatorname{MDV}_{\alpha}=\left[\begin{array}{c}
\left(\mathrm{m}_{0}\right) \\
\left(\mathrm{m}_{1)}\right. \\
\vdots \\
\left(\mathrm{m}_{\mathrm{n}}\right)
\end{array}\right] \quad \sum_{\mathrm{i}=0}^{\mathrm{n}} \mathrm{m}_{\mathrm{i}}=1
$$

365 where $m_{0}$ is the fractional abundance of molecules with mono-isotopic mass and $m_{i>0}$ is 366 the abundance of fragments with heavier masses. The GC-MS data were corrected for 367 the naturally occurring isotopes of oxygen $(\mathrm{O})$, hydrogen $(\mathrm{H})$ and carbon $(\mathrm{C})$ atoms using 368 a correction matrix (Eq. 2) as described by Nanchen et al ${ }^{45}$.

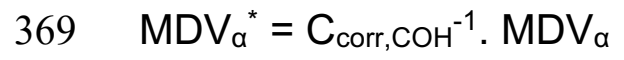

370 where $M D V_{\alpha}{ }^{*}$ is the corrected mass isotopomer distribution vector and $C_{\mathrm{corr}, \mathrm{COH}^{-1}}$ is the 371 correction matrix. According to Equation 3, the resulting $M D V_{\alpha}{ }^{*}$ values were then used to 372 assess the fractional labeling (FL) of amino acids whose carbon skeletons are derived 373 from their precursors in the central carbon metabolism.

$374 \quad \mathrm{FL}=\frac{\sum_{i=0}^{n} i \cdot m i}{n \cdot \sum_{i=0}^{n} m i}$

375 where $n$ represents the number of carbon atoms in the amino acid and $i$ is the mass 376 isotopomer. Corrected MDV for seven proteinogenic amino acids is shown in Table S1.

NMR samples were prepared by spiking neat solution with 50 microliters of $\mathrm{D}_{2} \mathrm{O}$ 378 with a 10x concentrated solution of Phosphate buffer and TMSP (Sodium-3379 Trimethylsilylpropinoate-d4, Cambridge Isotopes), for a final solution of 550 microliters, $38070 \mathrm{mM}$ Phosphate buffer and $0.91 \mathrm{mM}$ TMSP as an internal chemical shift and 381 concentration standard. All ${ }^{1} \mathrm{H}$ NMR experiments were collected on a $600 \mathrm{MHz}$ Bruker 382 Avance III NMR spectrometer equipped with a Bruker $5 \mathrm{~mm} \mathrm{1H/X} \mathrm{broadband} \mathrm{probe} \mathrm{with}$ 383 sample temperature controlled at $25^{\circ} \mathrm{C}$. Acquisition parameters were as follows: the 1D 384 NOESY-presaturation experiment was used (Bruker pulse program noesypr1d) with a 385 water presaturation pulse equivalent to $12 \mathrm{~Hz}$ field strength during both a 5 second 
386 relaxation delay and during a 50 millisecond NOESY mixing time. Data was collected with 387 a 20 ppm spectral window, 256 scans with 8 dummy scans, and $128 \mathrm{k}$ acquired points 388 equivalent to 5.5 seconds of acquisition time. All spectra were processed using 389 MestreNova version 14, which included $0.2 \mathrm{~Hz}$ exponential line-broadening before Fourier 390 transform, manual phase correction, polynomial baseline correction, and chemical shift 391 referencing to TMSP at $0.0 \mathrm{ppm}$. To obtain quantifications and isotopomer ratios, ${ }^{1} \mathrm{H}$ 392 spectral deconvolution was performed using the MestreNova Line Fitting tool. Peak areas 393 were exported to Microsoft Excel for further analysis.

\section{References}

396

3971 Nichols, E. M. et al. Hybrid bioinorganic approach to solar-to-chemical conversion. Proc Natl Acad Sci U S A 112, 11461-11466 (2015).

3992 Liu, C., Colón, B. C., Ziesack, M., Silver, P. A. \& Nocera, D. G. Water splitting400 biosynthetic system with $\mathrm{CO}_{2}$ reduction efficiencies exceeding photosynthesis. $401 \quad$ Science 352, 1210-1213 (2016).

4023 Sahoo, P. C., Pant, D., Kumar, M., Puri, S. K. \& Ramakumar, S. S. V. Material403 Microbe Interfaces for Solar-Driven $\mathrm{CO}_{2}$ Bioelectrosynthesis. Trends in $404 \quad$ Biotechnology(2020).

4054 Hohmann-Marriott, M. F. \& Blankenship, R. E. Evolution of Photosynthesis. Annual $406 \quad$ Review of Plant Biology 62, 515-548 (2011).

4075 Nugent, J. H. A. Oxygenic Photosynthesis. European Journal of Biochemistry 237, $408 \quad 519-531(1996)$.

4096 Georgianna, D. R. \& Mayfield, S. P. Exploiting diversity and synthetic biology for $410 \quad$ the production of algal biofuels. Nature 488, 329 (2012).

4117 Rosenbaum, M., He, Z. \& Angenent, L. T. Light energy to bioelectricity: 412 photosynthetic microbial fuel cells. Current Opinion in Biotechnology 21, 259-264 413 (2010). 
4148 Nozzi, N. E., Oliver, J. W. K. \& Atsumi, S. Cyanobacteria as a Platform for Biofuel $415 \quad$ Production. Frontiers in bioengineering and biotechnology 1, 7-7 (2013).

4169 Xiong, W. et al. The plasticity of cyanobacterial metabolism supports direct $\mathrm{CO}_{2}$ 417 conversion to ethylene. Nature Plants 1, 15053 (2015).

41810 Lan, E. I. \& Liao, J. C. ATP drives direct photosynthetic production of 1-butanol in 419 cyanobacteria. Proceedings of the National Academy of Sciences 109, 6018-6023 $420 \quad$ (2012).

42111 Kusakabe, T. et al. Engineering a synthetic pathway in cyanobacteria for isopropanol production directly from carbon dioxide and light. Metabolic Engineering 20, 101-108 (2013).

42412 Schirmer, A., Rude, M. A., Li, X., Popova, E. \& del Cardayre, S. B. Microbial $425 \quad$ Biosynthesis of Alkanes. Science 329, 559-562 (2010).

42613 Singh, A. K. \& Mallick, N. Advances in cyanobacterial polyhydroxyalkanoates production. FEMS Microbiology Letters 364(2017).

42814 Pospíšil, P. Production of reactive oxygen species by photosystem II. Biochimica et Biophysica Acta (BBA) - Bioenergetics 1787, 1151-1160 (2009).

43015 Dogutan, D. K. \& Nocera, D. G. Artificial Photosynthesis at Efficiencies Greatly Exceeding That of Natural Photosynthesis. Accounts of Chemical Research 52, 3143-3148 (2019).

43316 Nocera, D. G. The Artificial Leaf. Accounts of Chemical Research 45, 767-776 434 (2012).

43517 Lewis, N. S. Research opportunities to advance solar energy utilization. Science $436 \quad 351$, aad1920 (2016).

43718 Cook, T. R. et al. Solar Energy Supply and Storage for the Legacy and Nonlegacy $438 \quad$ Worlds. Chemical Reviews 110, 6474-6502 (2010).

43919 Pisciotta, J. M., Zou, Y. \& Baskakov, I. V. Light-dependent electrogenic activity of 440 cyanobacteria. PLoS One 5, e10821-e10821 (2010). 
44120 Tanaka, K., Kashiwagi, N. \& Ogawa, T. Effects of light on the electrical output of 442 bioelectrochemical fuel-cells containing Anabaena variabilis M-2: Mechanism of the post-illumination burst. Journal of Chemical Technology \& Biotechnology $\mathbf{4 2}$, 235-240 (1988).

44521 Torimura, M., Miki, A., Wadano, A., Kano, K. \& Ikeda, T. Electrochemical investigation of cyanobacteria Synechococcus sp. PCC7942-catalyzed photoreduction of exogenous quinones and photoelectrochemical oxidation of water. Journal of Electroanalytical Chemistry 496, 21-28 (2001).

44922 Sawa, M. et al. Electricity generation from digitally printed cyanobacteria. Nat Commun 8, 1327-1327 (2017).

45123 Levy, P. F., Sanderson, J. E., Kispert, R. G. \& Wise, D. L. Biorefining of biomass to liquid fuels and organic chemicals. Enzyme and Microbial Technology 3, 207215 (1981).

45424 Steinbusch, K. J. J., Hamelers, H. V. M., Plugge, C. M. \& Buisman, C. J. N. Biological formation of caproate and caprylate from acetate: fuel and chemical production from low grade biomass. Energy Environ. Sci. 4, 216-224 (2011).

45725 Holtzapple, M. T. et al. in Twentieth Symposium on Biotechnology for Fuels and 458 Chemicals (eds Davison B.H. \& Finkelstein M.) Ch. Chapter 56, 609-631 (Humana Press, 1999).

46026 Trebst, A. in Methods in Enzymology Vol. 69 (ed Anthony San Pietro) 675-715 $461 \quad$ (Academic Press, 1980).

46227 Vermaas, W. F. J., Williams, J. G. K., Rutherford, A. W., Mathis, P. \& Arntzen, C. J. Genetically engineered mutant of the cyanobacterium Synechocystis 6803 lacks the photosystem II chlorophyll-binding protein CP-47. Proceedings of the National Academy of Sciences 83, 9474-9477 (1986).

46628 Zhu, C., Li, H., Fu, S., Du, D. \& Lin, Y. Highly efficient nonprecious metal catalysts towards oxygen reduction reaction based on three-dimensional porous carbon nanostructures. Chem Soc Rev 45, 517-531 (2016). 
46929 Saar, K. L. et al. Enhancing power density of biophotovoltaics by decoupling storage and power delivery. Nature Energy 3, 75-81 (2018).

$47130 \quad$ Mackay, S. P. \& O 'Malley, P. J. Molecular Modelling of the Interaction between

$472 \quad$ DCMU and the $Q_{B}$-Binding Site of Photosystem II. Zeitschrift für Naturforschung C $473 \quad 48,191-198(1993)$.

47431 Roberts, A. G., Bowman, M. K. \& Kramer, D. M. The inhibitor DBMIB provides 475 insight into the functional architecture of the Qo site in the cytochrome $b 6 f$ complex. $476 \quad$ Biochemistry 43, 7707-7716 (2004).

47732 Honeycutt, R. C. \& Krogmann, D. W. Inhibition of chloroplast reactions with phenylmercuric acetate. Plant Physiol 49, 376-380 (1972).

47933 Aizawa, K. Carbonic anhydrase and $\mathrm{CO}_{2}$ concentrating mechanisms in microalgae and cyanobacteria. FEMS Microbiology Letters 39, 215-233 (1986).

48134 Hambourger, M. et al. Biology and technology for photochemical fuel production. Chem Soc Rev 38, 25-35 (2009).

$483 \quad 35$ Blankenship, R. E. et al. Comparing photosynthetic and photovoltaic efficiencies and recognizing the potential for improvement. Science 332, 805-809 (2011).

48536 Wittmiß, M., Mikkat, S., Hagemann, M. \& Bauwe, H. Stoichiometry of two plant glycine decarboxylase complexes and comparison with a cyanobacterial glycine cleavage system. The Plant Journal 103, 801-813 (2020).

48837 Hanna, M. C. \& Nozik, A. J. Solar conversion efficiency of photovoltaic and photoelectrolysis cells with carrier multiplication absorbers. Journal of Applied Physics 100, 074510 (2006).

49138 Foyer, C. H., Bloom, A. J., Queval, G. \& Noctor, G. Photorespiratory Metabolism: 492 Genes, Mutants, Energetics, and Redox Signaling. Annual Review of Plant Biology $493 \quad 60,455-484(2009)$.

49439 Zhu, X.-G., Long, S. P. \& Ort, D. R. Improving Photosynthetic Efficiency for Greater 495 Yield. Annual Review of Plant Biology 61, 235-261 (2010). 
49640 Yamori, W. \& Shikanai, T. Physiological Functions of Cyclic Electron Transport 497 Around Photosystem I in Sustaining Photosynthesis and Plant Growth. Annual $498 \quad$ Review of Plant Biology 67, 81-106 (2016).

49941 Williams, J. G. K. in Methods in Enzymology Vol. 167 766-778 (Academic Press, $500 \quad$ 1988).

50142 Murakami, A., Kim, S. J. \& Fujita, Y. Changes in photosystem stoichiometry in

43 Murakami, A. \& Fujita, Y. Regulation of Stoichiometry between PSI and PSII in

44 Xiong, W., Liu, L., Wu, C., Yang, C. \& Wu, Q. 13C-tracer and gas chromatography-

45 Nanchen, A., Fuhrer, T. \& Sauer, U. Determination of metabolic flux ratios from 13C-experiments and gas chromatography-mass spectrometry data: protocol and principles. Methods Mol Biol 358, 177-197 (2007).

\section{Acknowledgments}

516 This work was authored in part by the National Renewable Energy Laboratory, operated 517 by Alliance for Sustainable Energy, LLC, for the U.S. Department of Energy (DOE) under 518 Contract No. DE-AC36-08G028308. Funding provided by a Laboratory Directed 519 Research and Development seed project at the National Renewable Energy Laboratory. 520 The views expressed in the article do not necessarily represent the views of the DOE or 521 the U.S. Government. The U.S. Government retains and the publisher, by accepting the 522 article for publication, acknowledges that the U.S. Government retains a nonexclusive, 523 paid-up, irrevocable, worldwide license to publish or reproduce the published form of this work, or allow others to do so, for U.S. Government purposes. We acknowledge Dr. Wim 
525 Vermaas at Arizona State University for providing a Synechocystis PSII deficient mutant. 526 We also thank Dr. Ji Hao for assistance on transport measurements.

527 Author contributions: W.X., J.B., D.S. project conception. Z.L., X.C., D.S., J.B., W.X. 528 designed, performed, and/or analyzed photo-electrochemical experiments. Z.L., C.W., 529 X.G., W.X. designed, performed, and/or analyzed biological experiments including 530 cyanobacteria cultivation, spectroscopic analysis, metabolite analysis, ${ }^{13} \mathrm{C}$-labeling and 531 GC-MS. B.A. performed and analyzed NMR data. Z.L., W.X., J.B. wrote the manuscript 532 with input from all authors and revisions from D.S. and J.Y.

533 Competing interests: Authors declare no competing interests. 


\section{Figures and figure legends}

Figure 1

a)

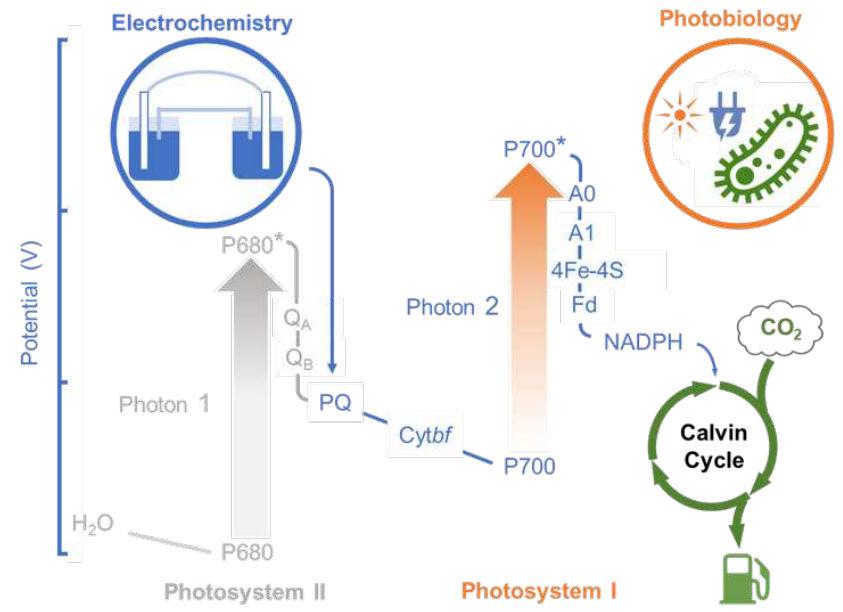

b)
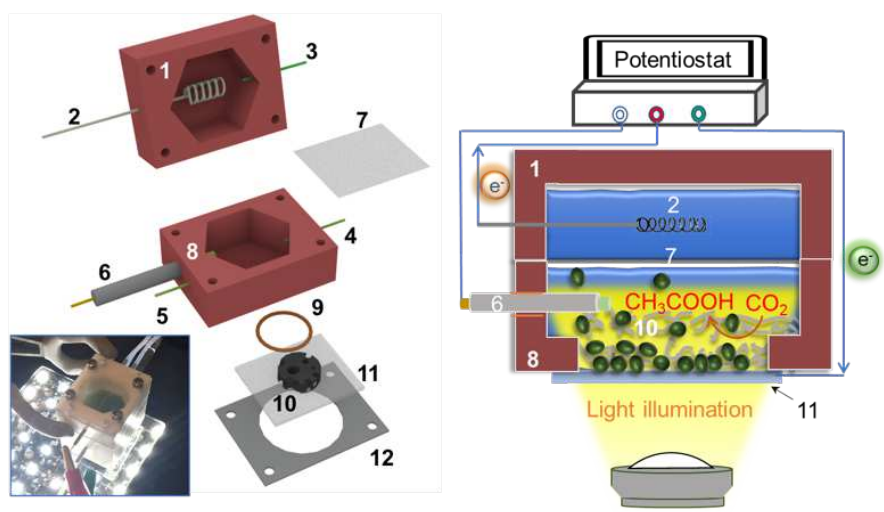

538 Fig. 1. Electrophototrophic system. a) The electrophototroph is designed for $\mathrm{CO}_{2}$-to-

539 fuels conversion with external supply of light and electricity to a tailored photosynthetic

540 microbe. To this end, photosystem II in natural photosynthesis (gray) can be genetically

541 removed, and instead the external electrochemical circuit delivers high-energy electrons

542 to photoexcited photosystem I (oxidized P700), and ultimately produces NADPH to drive

$543 \mathrm{CO}_{2}$ fixation. This process could leverage electron acceptors in the PETC including 544 plastoquinone $(P Q)$, Cytochrome b6f complex (Cytbf), special chlorophyl $\left(A_{0}\right)$, vitamin $K$ $545\left(\mathrm{~A}_{1}\right)$, iron-sulfur centers (4Fe-4S), and ferredoxin ( $\mathrm{fd}$ ) etc. Protons can be pumped across 546 the thylakoid membrane establishing a proton-motive force that can be used for the 547 synthesis of ATP. b) Schematic illustration and photograph (inset of left panel) of 
548 electrochemical device to shuttle electrons to PSII deficient cyanobacteria. Component 1 549 and 8: PTFE anodic part and cathodic part; Component 2: Platinum counter electrode; 550 Component 3, 4, 5: medium inlet/outlet; Component 6: $\mathrm{Ag} / \mathrm{AgCl}$ reference electrode; 551 Component 7: Nafion Membrane; Component 9: Seal O-ring; Component 10: Carbon felt; 552 Component 11: FTO glass; Component 12: Working electrode clamp. Right panel shows 553 the loading of cyanobacterial cells and the electrons delivery process. 
a)
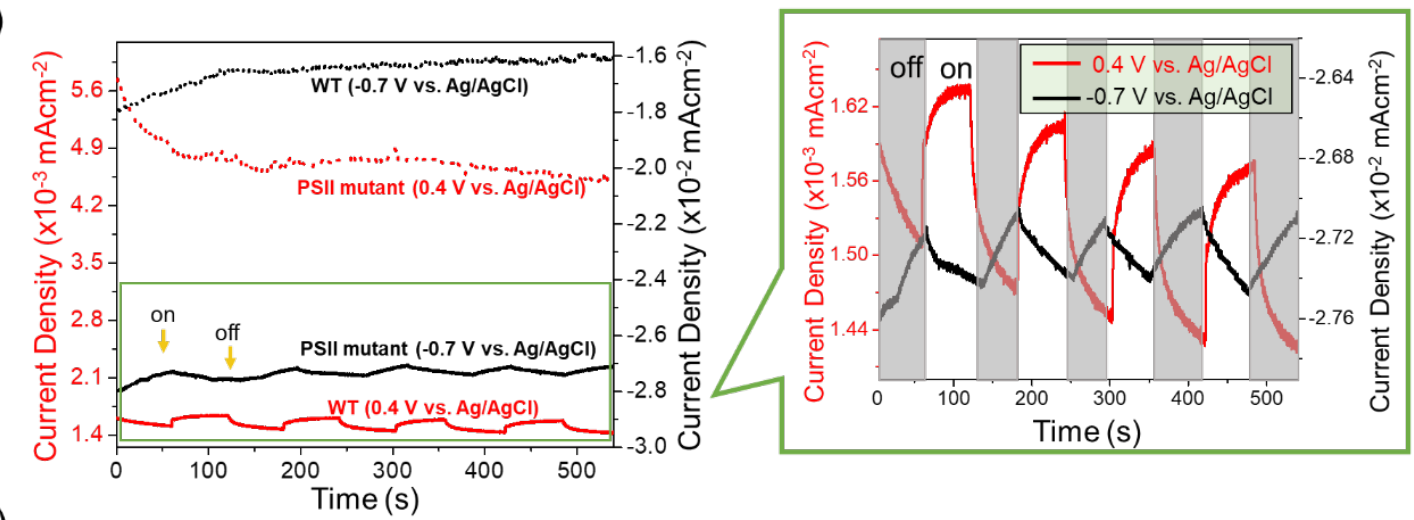

b)
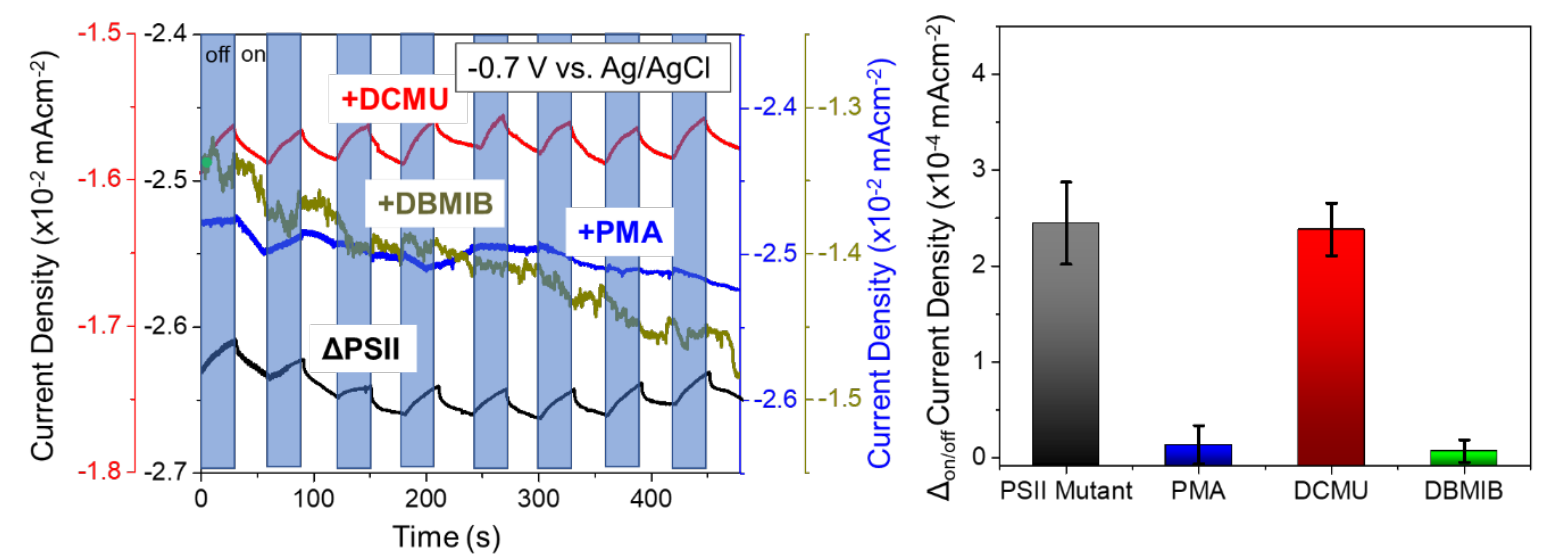

Fig. 2. Electrochemical properties of the electrophototrophic hybrid. a) i-t measurement under chopped illumination when cyanobacterial cells (Synechocystis WT 559 and $\triangle$ PSII mutant) were applied as electron donor (on anode, red solid and dash line) or 560 acceptor (on cathode, black solid and dash line). Right panel comparing the light561 response of WT and $\triangle$ PSIl cells as electron donor and acceptor, respectively. b) Current 562 density changes in response to light/dark switch with addition of site-specific PETC 563 inhibitors to cathodal $\triangle P S I$ culture. The time-course and differences of current density 564 between light and dark are shown on the left and right panel, respectively. 
a)

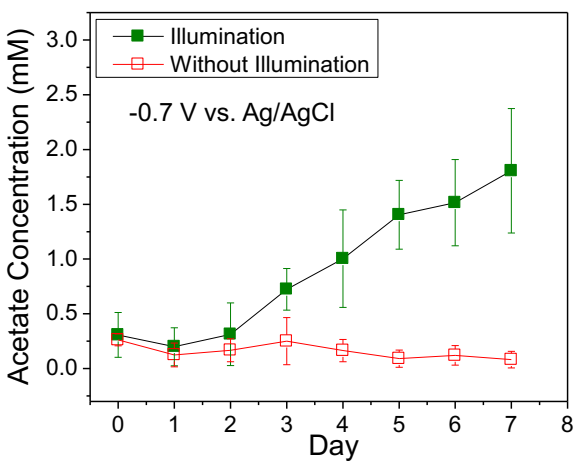

b)

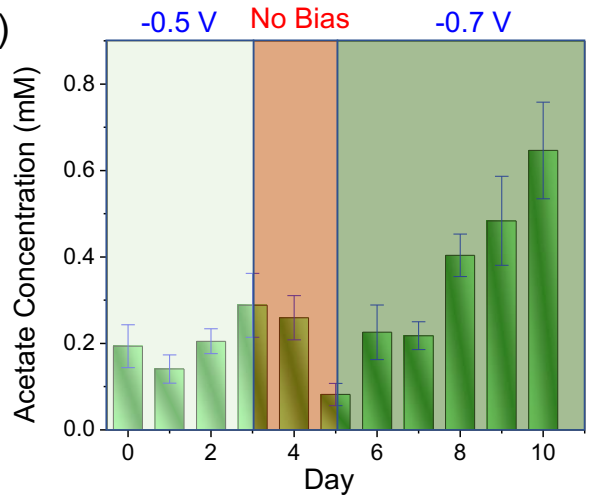

d)

c)

$\mathrm{CH}_{3} \mathrm{COOH}$
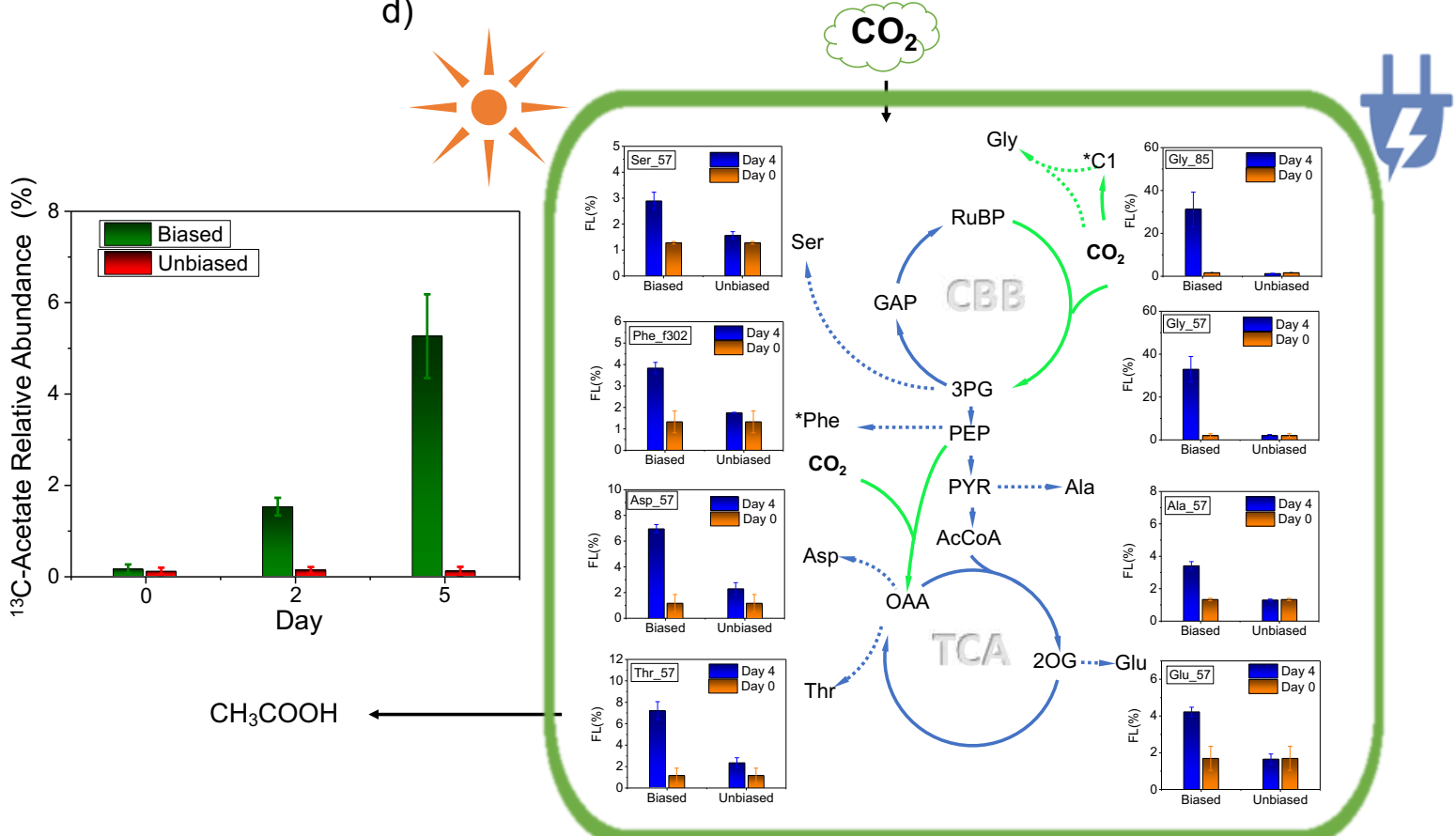

$\mathrm{CO}_{2}$

567 Fig. 3. $\mathbf{C O}_{2}$ valorization by the electrophototrophic hybrid. a) Electrophototrophic acetate production of $\triangle \mathrm{PSIl}$ by external electrical bias $(-0.7 \mathrm{~V}$ vs. $\mathrm{Ag} / \mathrm{AgCl})$ with and without illumination. b) Time course of electrophototrophic productivity for $\triangle \mathrm{PSI}$ under

570 illumination with different electron supplies. Error bars represent standard deviations from

571 biological triplicates. c) ${ }^{13} \mathrm{C}$-acetate production via fixation of ${ }^{13} \mathrm{CO}_{2}$ (derived from ${ }^{13} \mathrm{C}$ -

572 bicarbonate) in illuminated $\triangle$ PSII, with or without application of external electrical bias (-

$5730.7 \mathrm{~V}$ vs. $\mathrm{Ag} / \mathrm{AgCl}$ ). d) Fractional labeling of seven protein-bound amino acids that were

574 directly produced from the central carbon metabolism via fixation of ${ }^{13} \mathrm{CO}_{2}$ (derived from

$575{ }^{13} \mathrm{C}$-bicarbonate) in illuminated $\triangle \mathrm{PSI}$, with or without application of external electrical bias 
$576(-0.7 \mathrm{~V}$ vs. $\mathrm{Ag} / \mathrm{AgCl})$. Green arrows indicate $\mathrm{CO}_{2}$-fixing reactions. Dash lines are reactions 577 for the synthesis of amino acids. Abbreviations: RuBP, ribulose 1,5-bisphosphate; GAP, 578 glyceraldehyde 3-phosphate; 3PG, 3-phosphoglycerate; PEP, phosphoenolpyruvate; 579 PYR, pyruvate; AcCoA, acetyl coenzyme A; OAA, oxaloacetate; 2OG, 2-oxoglutarate. 580 CBB, the Calvin-Benson-Bassham Cycle; TCA, the tricarboxylic acid cycle. Amino acids 581 are presented by their 3-letter abbreviations. ${ }^{*}$ Phe indicates partial carbons of 582 phenylalanine (C1-3) synthesized from PEP. ${ }^{*} \mathrm{C} 1$ indicates that one carbon unit is the 583 precursor of glycine's methylene group and can be represented by the Gly_85 fragment 584 in GC-MS. 


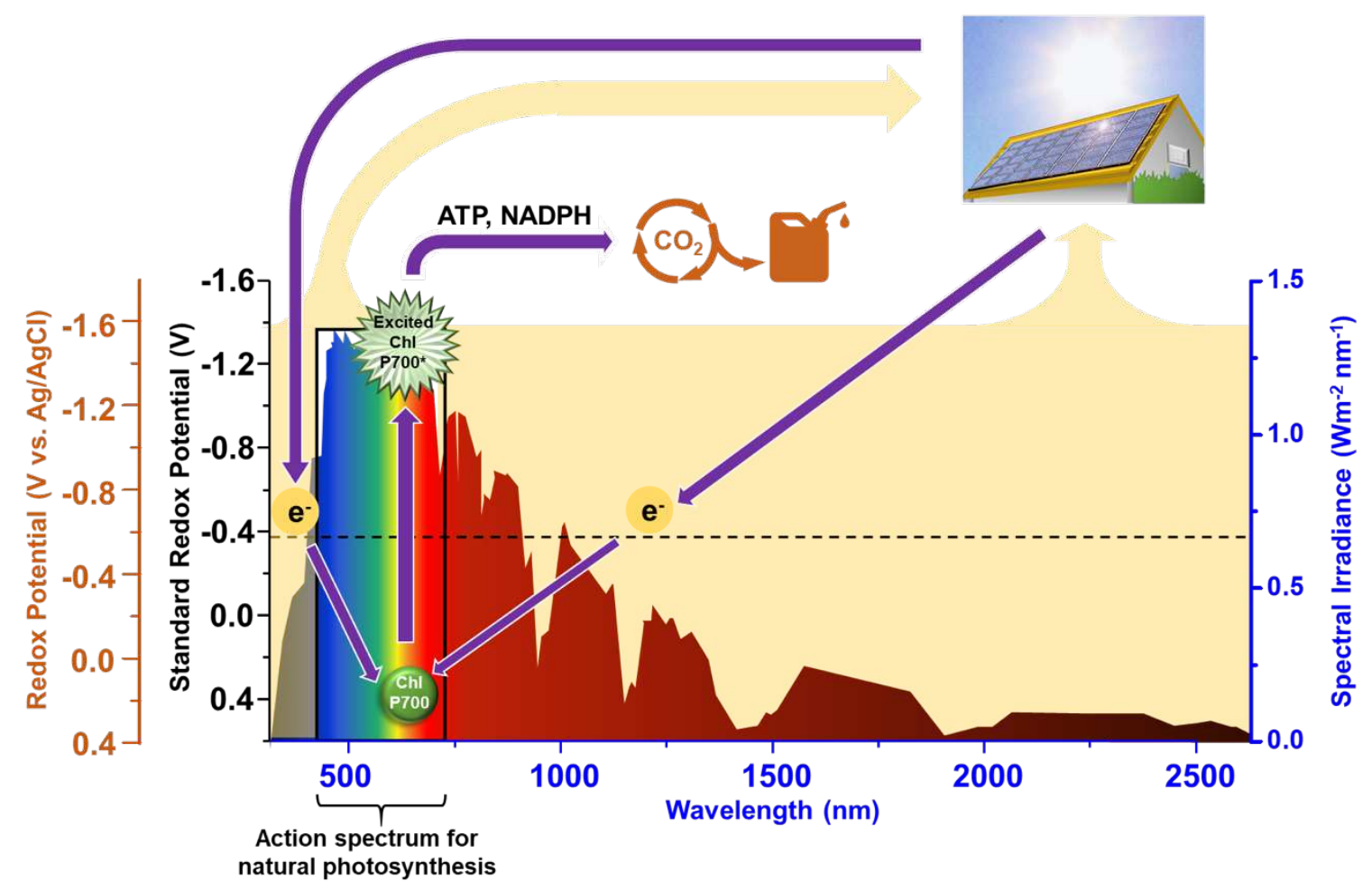

587 Fig. 4 Schematic illustration of a hybrid photosynthesis system to convert broader 588 spectrum of PAR than natural photosynthesis. Leveraging PV device, UV-blue and IR 589 regions that cannot be utilized by photosynthetic organisms, may be converted to 590 electricity to energize electrophototrophic cyanobacteria with single PSI for $\mathrm{CO}_{2}$ 591 valorization. Dash line represents the standard redox potential where the exocellular 592 electrons shuttled to the photosynthetic cells carrying a single photosystem. 


\section{Figures}

a)

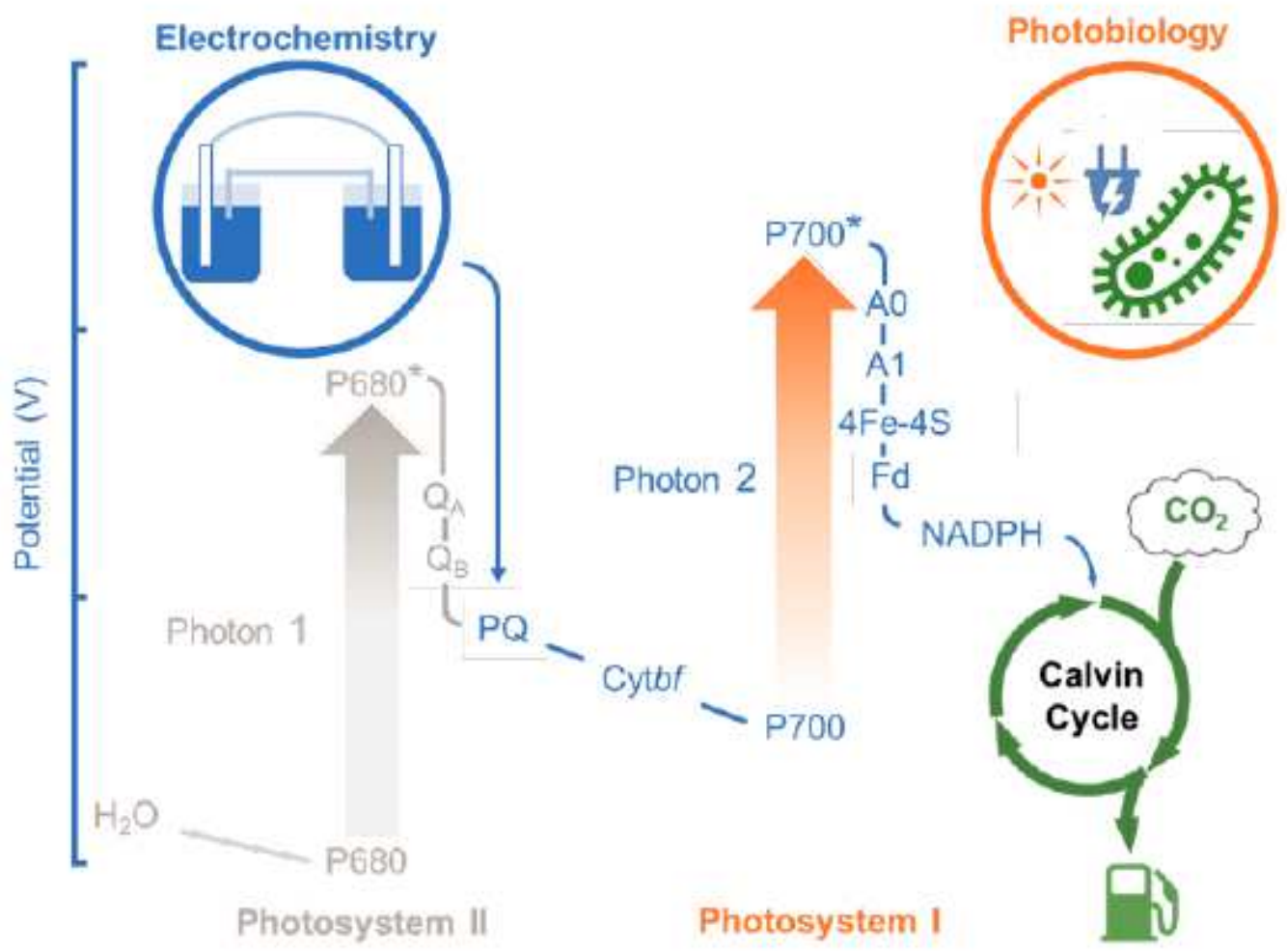

b)
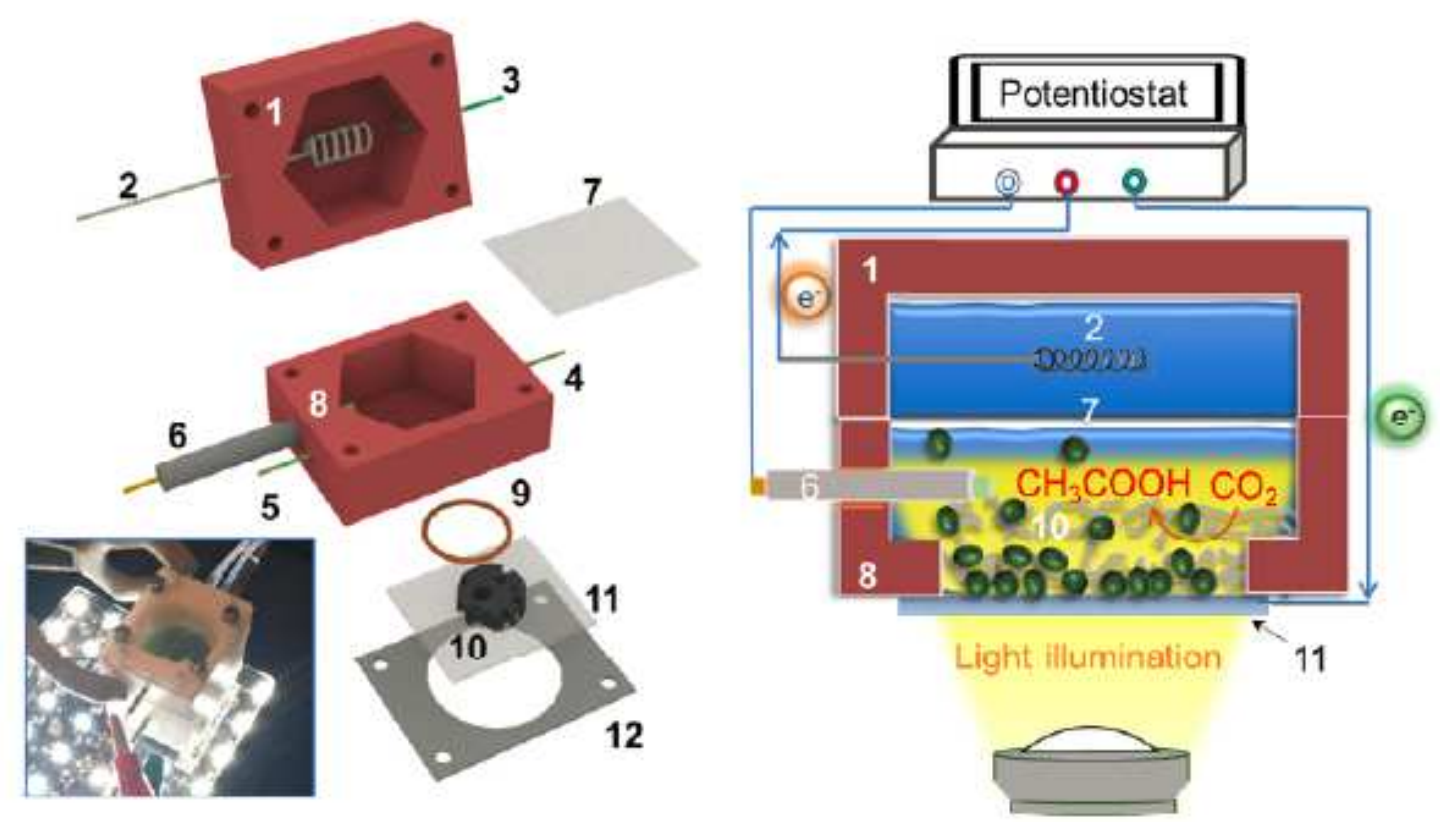

Figure 1

Electrophototrophic system. a) The electrophototroph is designed for CO2-to539 fuels conversion with external supply of light and electricity to a tailored photosynthetic microbe. To this end, photosystem II in natural photosynthesis (gray) can be genetically removed, and instead the external electrochemical circuit 
delivers high-energy electrons to photoexcited photosystem I (oxidized P700), and ultimately produces NADPH to drive $\mathrm{CO} 2$ fixation. This process could leverage electron acceptors in the PETC including plastoquinone (PQ), Cytochrome b6f complex (Cytbf), special chlorophyl ( $A 0)$, vitamin $K(A 1)$, iron-sulfur centers (4Fe-4S), and ferredoxin ( $\mathrm{fd}$ ) etc. Protons can be pumped across the thylakoid membrane establishing a proton-motive force that can be used for the synthesis of ATP. b) Schematic illustration and photograph (inset of left panel) of electrochemical device to shuttle electrons to PSII deficient cyanobacteria. Component 1 and 8: PTFE anodic part and cathodic part; Component 2: Platinum counter electrode; Component 3, 4, 5: medium inlet/outlet; Component 6: $\mathrm{Ag} / \mathrm{AgCl}$ reference electrode; Component 7: Nafion Membrane; Component 9: Seal O-ring; Component 10: Carbon felt; Component 11: FTO glass; Component 12: Working electrode clamp. Right panel shows the loading of cyanobacterial cells and the electrons delivery process.

a)
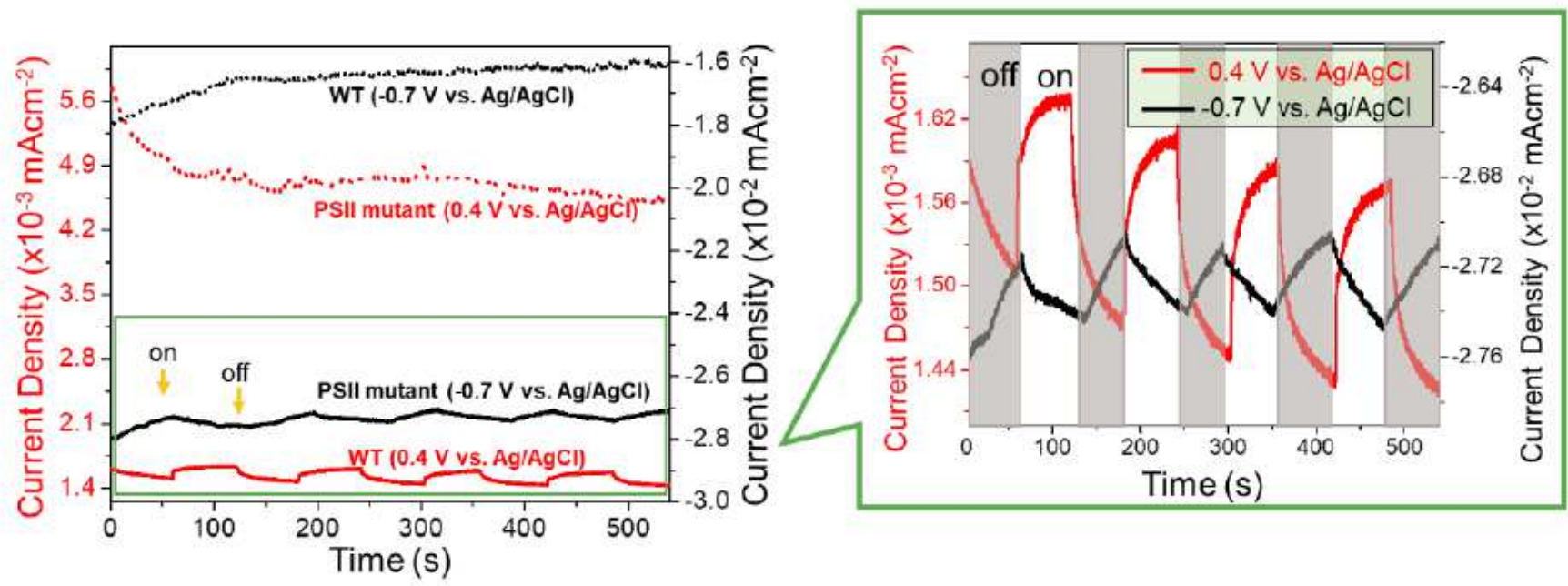

b)
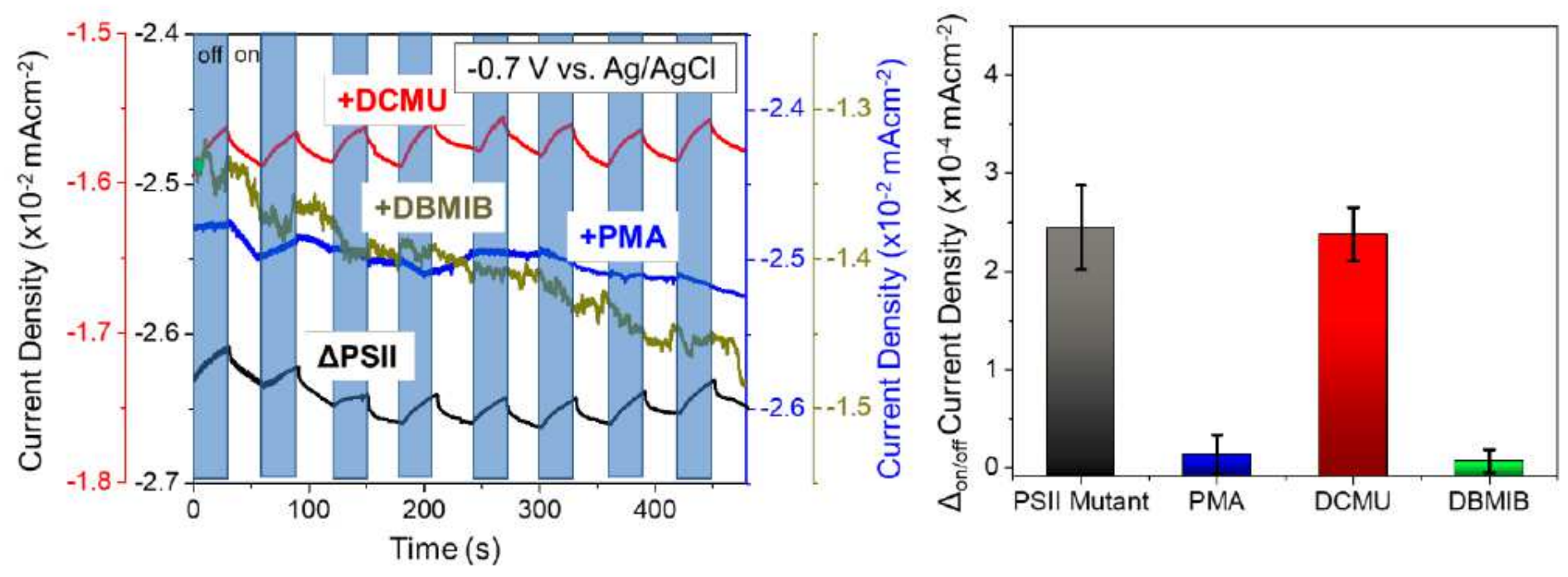

Figure 2

Electrochemical properties of the electrophototrophic hybrid. a) i-t measurement under chopped illumination when cyanobacterial cells (Synechocystis WT and $\triangle$ PSIl mutant) were applied as electron donor (on anode, red solid and dash line) or acceptor (on cathode, black solid and dash line). Right panel 
comparing the light response of WT and $\triangle \mathrm{PS}$ II cells as electron donor and acceptor, respectively. $\mathrm{b}$ ) Current density changes in response to light/dark switch with addition of site-specific PETC inhibitors to cathodal $\triangle \mathrm{PSIl}$ culture. The time-course and differences of current density between light and dark are shown on the left and right panel, respectively.

a)

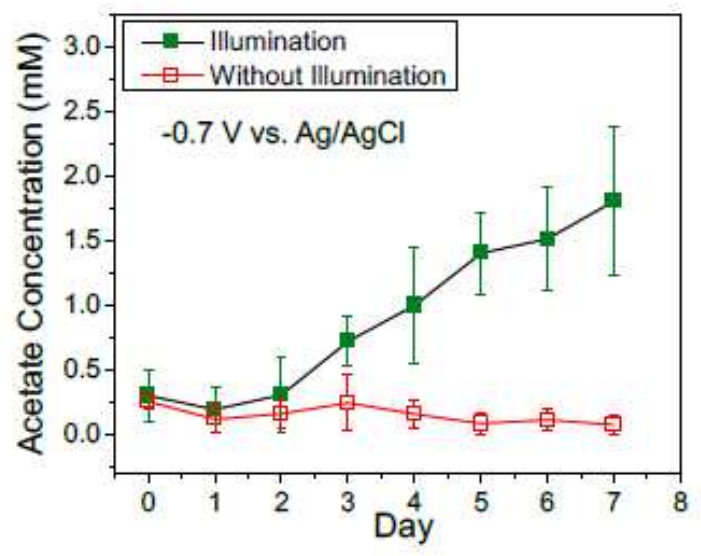

b)

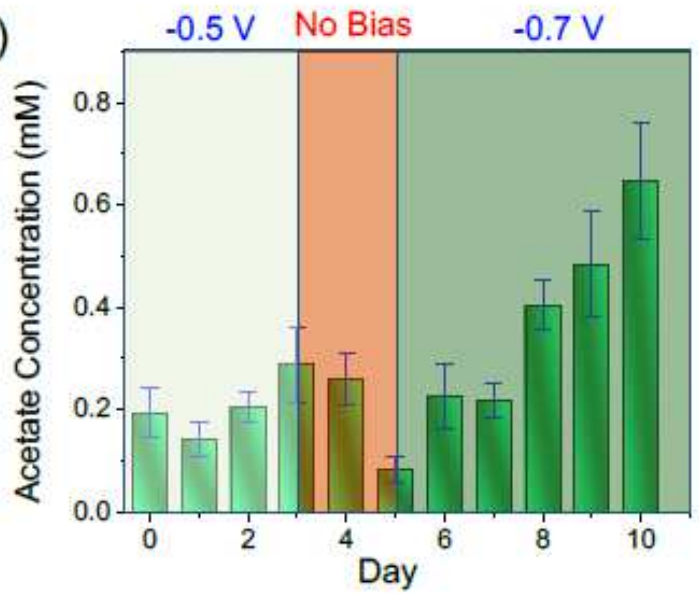

d)

c)

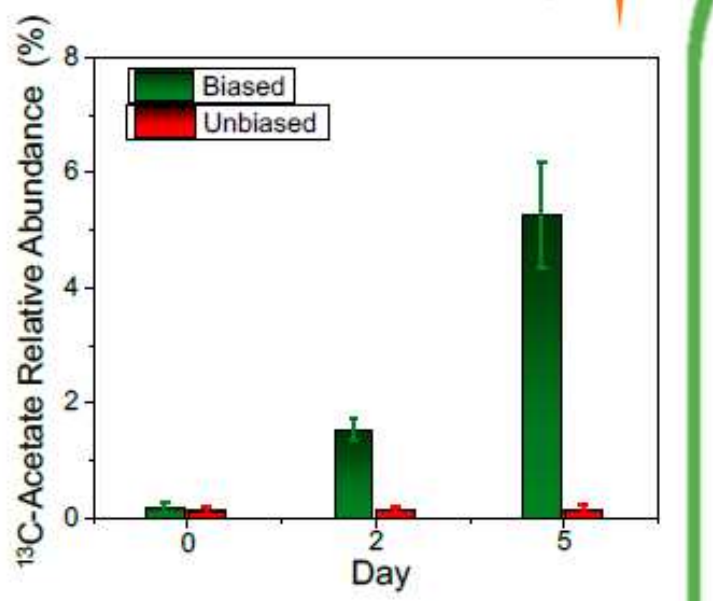

$\mathrm{CH}_{3} \mathrm{COOH}$

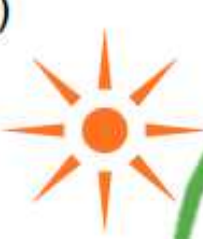

$\mathrm{CO}_{2}$

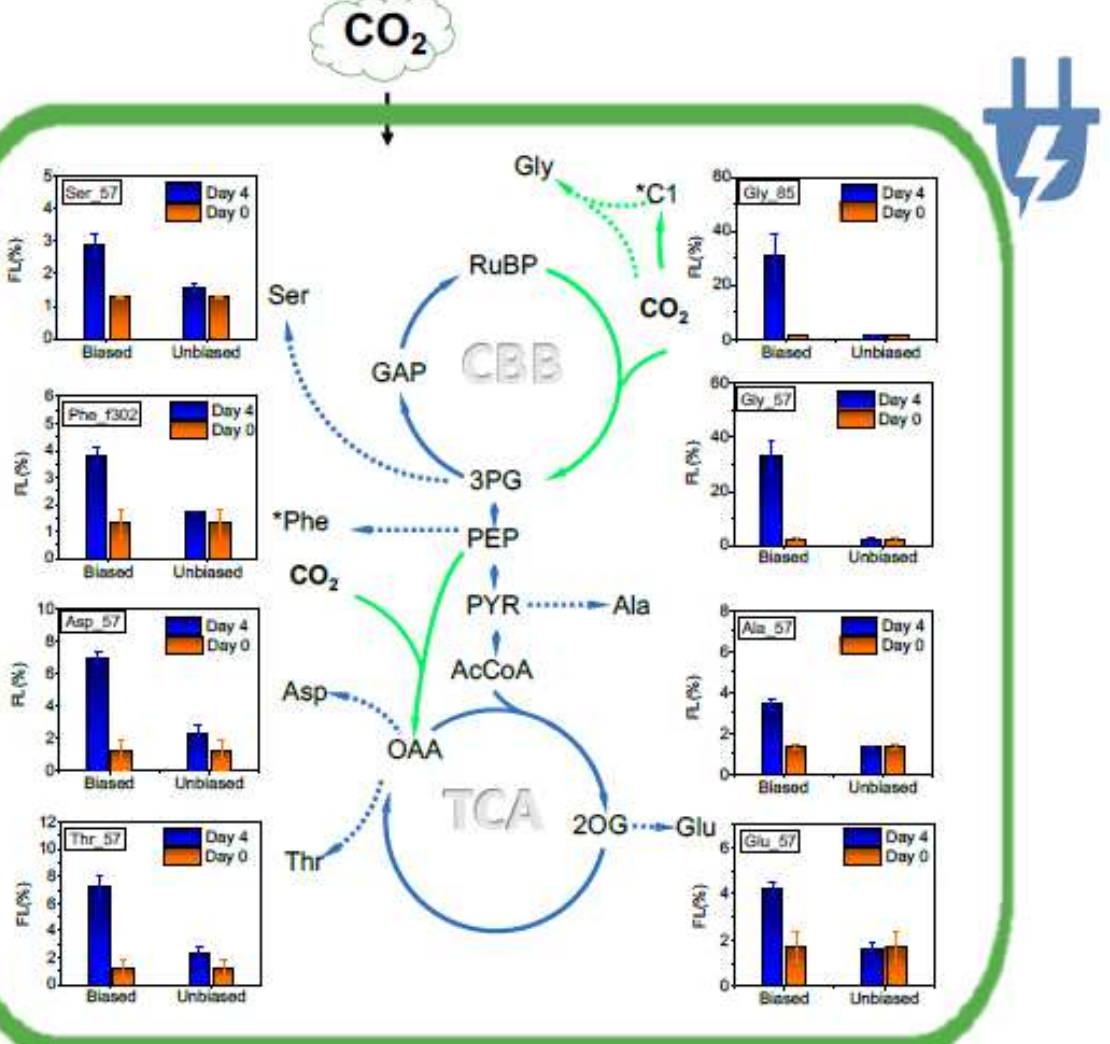

Figure 3

$\mathrm{CO} 2$ valorization by the electrophototrophic hybrid. a) Electrophototrophic acetate production of $\triangle \mathrm{PSII}$ by external electrical bias (-0.7 V vs. $\mathrm{Ag} / \mathrm{AgCl})$ with and without illumination. b) Time course of electrophototrophic productivity for $\triangle \mathrm{PSII}$ under illumination with different electron supplies. Error bars represent standard deviations from biological triplicates. c) 13C-acetate production via fixation of $13 \mathrm{CO} 2$ (derived from $13 \mathrm{C}$ bicarbonate) in illuminated $\triangle \mathrm{PSII}$, with or without application of external electrical bias 
$(-0.7 \mathrm{~V} v \mathrm{vs} . \mathrm{Ag} / \mathrm{AgCl})$. d) Fractional labeling of seven protein-bound amino acids that were directly produced from the central carbon metabolism via fixation of $13 \mathrm{CO} 2$ (derived from 13C-bicarbonate) in illuminated $\triangle \mathrm{PSII}$, with or without application of external electrical bias $(-0.7 \mathrm{Vvs}$. $\mathrm{Ag} / \mathrm{AgCl})$. Green arrows indicate $\mathrm{CO} 2$-fixing reactions. Dash lines are reactions for the synthesis of amino acids. Abbreviations: RuBP, ribulose 1,5-bisphosphate; GAP, glyceraldehyde 3-phosphate; 3PG, 3-phosphoglycerate; PEP, phosphoenolpyruvate; PYR, pyruvate; AcCoA, acetyl coenzyme A; OAA, oxaloacetate; 20G, 2-oxoglutarate. $\mathrm{CBB}$, the Calvin-Benson-Bassham Cycle; TCA, the tricarboxylic acid cycle. Amino acids are presented by their 3-letter abbreviations. *Phe indicates partial carbons of phenylalanine (C1-3) synthesized from PEP. ${ }^{*} \mathrm{C} 1$ indicates that one carbon unit is the precursor of glycine's methylene group and can be represented by the Gly_85 fragment in GC-MS.

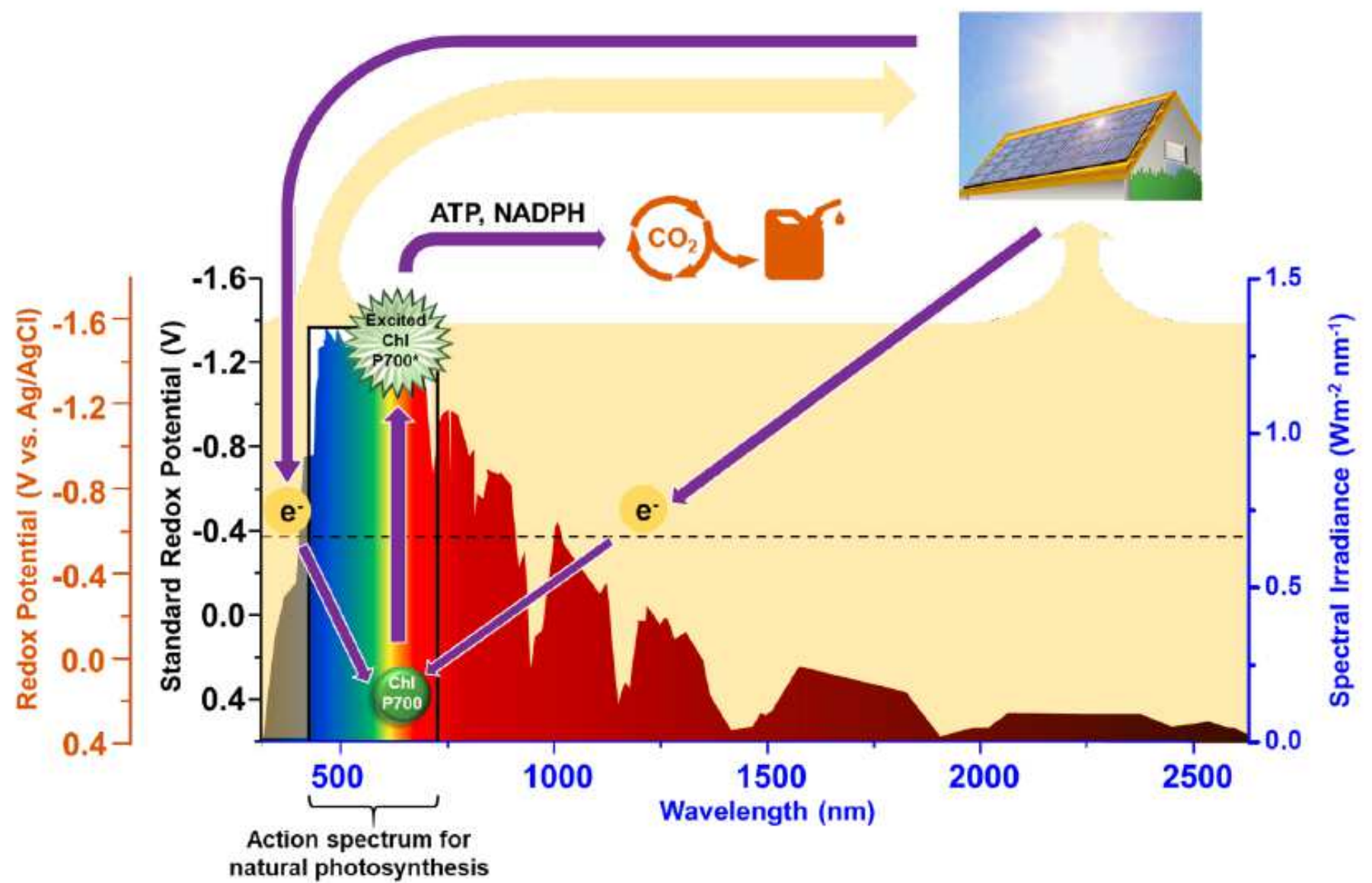

\section{Figure 4}

Schematic illustration of a hybrid photosynthesis system to convert broader spectrum of PAR than natural photosynthesis. Leveraging PV device, UV-blue and IR regions that cannot be utilized by photosynthetic organisms, may be converted to electricity to energize electrophototrophic cyanobacteria with single PSI for $\mathrm{CO} 2$ valorization. Dash line represents the standard redox potential where the exocellular electrons shuttled to the photosynthetic cells carrying a single photosystem.

\section{Supplementary Files}


This is a list of supplementary files associated with this preprint. Click to download.

- SupplementaryMaterials.pdf 